Monográfico: Reflujo Vesicoureteral

Arch. Esp. Urol., 61, 2 (349-353), 2008

\title{
REFLUJO VÉSICO URETERAL SINTOMÁTICO EN LA VIDA ADULTA. TESTIMONIO DE UNA PACIENTE
}

\author{
Carlos Miguélez Lago y Jairo Moreno Román.
}

Urología Pediátrica. Clínica Santa Elena. Torremolinos. Málaga. España.

Resumen.- OBJETIVO: Existe la opinión de que el Reflujo Vésico Ureteral (RVU) primario durante la vida adulta tiene pocas o nulas manifestaciones clínicas. Sin embargo es posible encontrar un RVU activo en esta época de la vida. Se presenta el caso de una paciente adulta que ilustra el concepto de "Reflujo Sintomático" y describe con sus propias palabras el síntoma del "Dolor de Reflujo". Estudiar la clínica del RVU sintomático en una adulta con repercusión en la calidad de vida.

MÉTODO/RESULTADO: Paciente de sexo femenino y 33 años de edad, con historia de pielonefritis crónica desde los 28 años, por lo que se diagnóstico RVU bilateral derecho grado III con duplicidad incompleta e izquierdo grado II, no conocido con anterioridad. No fue intervenida y le fue prescrita profilaxis antibiótica, a pesar de la cual presentó sintomatología clínica de 4 años de evolución con: Febrícula constante con frecuentes brotes de fiebre superior a 38ㅜ, pérdida de $8 \mathrm{Kg}$ de peso, mal estar general y sobre todo "Dolor de Reflujo" que la propia paciente describe como "agudo, intenso, lacerante, ascendente, localizado en el trayecto ureteral y celda renal, comenzando unos minutos antes y finalizando unos minutos después de la micción". Durante 4 años descartaron las posibles etiologías de un síndrome febril de origen desconocido, y al final fue remitida para tratamiento del RVU. Se hizo tratamiento endoscópico (TE) bilateral con dextranómero / Ácido Hialurónico estabilizado no animal, logrando el cese del RVU en la primera inyección. El dolor del Reflujo desapareció en los primeros días de postoperatorio y el síndrome febril antes del mes. No ha vuelto a recidivar la sintomatología durante 4 años de seguimiento.

CONCLUSIONES: El RVU puede ser sintomático en la vida adulta, con un dolor muy peculiar y fácil de identificar, además de un síndrome febril con deterioro progresivo. El TE puede curar el RVU, erradicar su sintomatología, y mejorar la calidad de vida.

Palabras clave: Reflujo Vésico Ureteral. Adultos. Pielonefritis Crónica. Dolor de Reflujo. Reflujo Sintomático. Tratamiento endoscópico.

Summary.- OBJECTIVES: There is a general opinion about that vesicoureteral reflux (VUR) rarely produces symptoms during adulthood. But it is possible to find active VUR over 20 years of age. A case report of a woman 28 years old with symptomatic VUR is presented with description of the "Reflux Pain" by herself. The objective of this article is to study the clinical aspects of symptomatic VUR in an adult woman with impairment in her quality of life.

METHODS/RESULTS: She was a 33 years old female patient, with chronic and febrile breakthrough urinary tract infections 
(UTI) since she was 28 years of age. Then an unknown bilateral VUR was diagnosed. It was grade III in an incompletely duplicated right side and grade II in the left one. She recived antibiotic prophylaxis (AP) for 4 years, in spite of it she had a temperature over 370, with frequent peaks over 380, and she lost $8 K G$ of weight. She also had lumbar pain and "Reflux Pain", which was described by the patient as: "acute, intense, excruciating, ascending, located in the ureters and kidneys, beginning some minutes before urinating and finishing some minutes later. During these 4 years many others pathologies were ruled out looking for other etiology of the temperature of "unknown origin". Finally she was sent to urology for treatment of VUR. Bilateral Endoscopic Treatment (ET) with non animal stabilized Hyaluronic acid/Dextranomer (DX/NASHA) gel was performed, with good result after the first injection. "Reflux Pain" disappeared few days after ET and after one month the temperature was under 37o. She remains asymptomatic after 4 years of follow up.

CONCLUSIONS: VUR can produce symptoms during adulthood, with a very typical pain easy to identify, chronic pyelonephritis with temperature and progressive deterioration.

ET can eliminate VUR, stop the symptoms and improve quality of life.

Keywords: Vesico Ureteral Reflux. Adults. Chronic Pyelonephritis. Reflux pain. Symptomatic Reflux. Endoscopic Treatment.

\section{INTRODUCCIÓN}

El RVU es una patología propia de la infancia, que puede ser congénita sobre todo en el sexo masculino con dilatación fetal del tracto urinario superior (1). Se conoce como RVU primario al RVU no producido por otra patología orgánica conocida como ureterocele, válvulas uretrales, uréter ectópico, vejiga neurógena, etcétera.

El RVU 1, sobre todo con Infección del Tracto Urinario (ITU) puede producir una Nefropatía de Reflujo (NR), más fácilmente durante la infancia (1).

Sin embargo el RVU también se puede diagnosticar por primera vez en adultos desde la juventud hasta la senectud, con una media de 30 años de edad (2-9). Se estima que el $15 \%$ de adultos con pielonefritis crónica y más del $25 \%$ de adultos con ITU recurrente tienen RVU $(10,11)$. Choi y cols. encuentran RVU en el $2.3 \%$ de mujeres adultas con pielonefritis aguda no complicada (12).

Existe una opinión bastante generalizada, de que el RVU $1^{\circ}$ durante la vida adulta tiene pocas manifestaciones clínicas.

Se presenta el caso de una paciente adulta que ilustra el concepto de reflujo sintomático y describe con sus propias palabras el síntoma del "dolor de reflujo".

\section{OBJETIVO}

Estudiar la clínica y semiología del RVU en la edad adulta, y su repercusión en la calidad de vida.

\section{CASO CLÍNICO}

Paciente de sexo femenino y 33 años de edad que consulta por RVU bilateral diagnosticado 5 años antes.

\section{Antecedentes}

El motivo del diagnóstico fue pielonefritis de repetición, desde los 28 años de edad.

Seis meses después se realizó estudio urológico con ecografía, urografía intravenosa, Cistouretrografía Miccional Seriada (CUMS) y gammagrafía renal que evidenció:

- Duplicidad pieloureteral derecha

- Cicatriz pielonefrítica en cáliz superior derecho

- Nefropatía de RVU derecha con disminución de tamaño y función

- RVU Derecho grado III a los 2 uréteres

- RVU Izquierdo grado II

No historia de RVU ni de pielonefritis previos.

Clínica de la paciente era:

- Febrícula $37.5^{\circ}$ casi permanente con picos de $38.5^{\circ}$

- Pérdida progresiva de peso desde 65 hasta $47 \mathrm{Kg}$.

- Mal estado general, decaimiento

- Dolor ascendente por recorrido ureteral hasta riñón, mayor en el lado derecho.

Las características del dolor fueron:

- Diario con frecuencia progresiva

- Mayor durante la micción

- Agudo, intenso y punzante

- Ascendente desde vejiga a uréteres y riñones.

- Duración aproximada 15-20 minutos

- Finalización 5-10 minutos después de terminar la micción.

Instauraron profilaxis antibiótica (PA), y la paciente fue estudiada como "fiebre de origen desconocido". Se descartaron otros diagnósticos incluidos procesos ginecológicos, cardiológicos, neoplásicos, paraneoplásicos, reumatológicos, enfermedades tropicales, etcétera, en diversos departamentos hospitalarios, pues inicialmente no se consideró al RVU como responsable de todo el cuadro clínico de la paciente.

No existía incontinencia urinaria ni clínica de disfunción vesical o esfinteriana.

\section{Exploración física}

Datos anómalos: afectación del estado general, peso de $47 \mathrm{Kg}$. para una talla de $1.73 \mathrm{~m}$, temperatura 37.5 , puño percusión renal derecha positiva.

La tensión arterial, auscultación cardio pulmonar, palpación abdominal y pélvica fueron normales. No existían edemas parpebrales ni de miembros inferiores. 


\section{Juicio Clínico}

Duplicidad pieloureteral derecha con RVU derecho grado III a los 2 uréteres e izquierdo grado II, nefropatía de reflujo derecha, pielonefritis crónica con deterioro general progresivo, síndrome febril mantenido, con intenso dolor reno-ureteral, de 5 años de evolución a pesar de la PA.

\section{Indicación Quirúrgica}

Se decide Tratamiento Endoscópico (TE) del RVU, en base a la larga evolución, la pielonefritis crónica, la clínica de fiebre y dolor, la edad y la nefropatía de reflujo derecha.

\section{Tratamiento Endoscópico}

Se realiza cistouretroscopia con sedación, siendo la uretra y cuello vesical normales.

Se encontró 1 meato ortotópico en cada lado, con escaso túnel submucoso y con hidrodistensión positiva. Con la hidrodistensión del meato derecho se vio un trayecto único de $2 \mathrm{~mm}$. dividiéndose a continuación en 2 uréteres formando una duplicidad pieloureteral derecha casi completa con meato común.

Con aguja metálica se inyectó Dextranómero/ Acido Hialurónico estabilizado no animal (DX/NASHA), 0.5 CC en cada lado.

Se administró Cefalosporina intravenosa de $3^{\mathrm{a}}$ generación al inicio del TE.

\section{Evolución, control y resultado postoperatorios}

- Se mantiene PA durante 3 meses de postoperatorio.

- Desaparición del dolor renoureteral desde los primeros días de postoperatorio.

- Desaparición progresiva de la fiebre, erradicándose totalmente un mes después del TE.

- Ecografía al mes sin evidencia de dilatación urétero-pielocalicial.

- CUMS a los 3 meses normal sin RVU ni residuo postmiccional.

- Seguimiento de 3 años sin infecciones urinarias ni pielonefritis ni dolor renoureteral. Aumento progresivo de peso hasta $61 \mathrm{Kg}$. , incorporación a la vida laboral, embarazo y parto normales.

\section{TESTIMONIO DE LA PACIENTE}

Se reproduce a continuación una descripción de la propia paciente de su situación clínica desde el inicio de su sintomatología hasta la desaparición de la misma, que ella escribió a petición de los autores, para reflejar con sus palabras la realidad subjetiva y objetiva de su proceso.

Fuí diagnosticada de duplicidad pielo ureteral derecha con un reflujo vesicoureteral bilateral: derecho superior e inferior de grado III en ambos uréteres y el izquierdo de grado II, que ha sido corregido mediante una sencilla intervención no sin antes pasar por numerosas pruebas y descartar también un larga lista de enfermedades antes de llegar a un diagnóstico claro, que paso a relatar a continuación:
"Todo comenzó cuando tenía 28 años, que fue la primera vez que sentí un dolor de riñón que resultó ser la primera pielonefritis, que se trató con antibióticos. A los 6 meses de la $1^{\underline{a}}$ sufrí otra pielonefritis, con un dolor intenso en el riñón derecho acompañado de fiebre alta que no le deseo a nadie. Fue tratada con más antibióticos que luego me dejaron una semana postrada.

Después de varios análisis de orina y cultivos positivos, tratados con antibióticos, me remitieron al Servicio de Urología de un Hospital por tener múltiples infecciones de orina que se van intensificando. Entonces me hicieron más pruebas:

- una urografía en la que se diagnosticó la duplicidad pieloureteral del riñón derecho, cáliz pielonefrítico y cicatriz cortical,

- una CUMS en el que aparece el reflujo vesicoureteral grado III a los dos uréteres derechos y grado II al izquierdo

- y una gammagrafía renal en la que se evidencia que el riñón derecho está dañado y tiene su función levemente disminuida.

- También me hacen un estudio ginecológico a instancias de los urólogos, que resulta normal, sin ninguna alteración.

Al final lo que se decide en sesión clínica es darme una terapia con antibióticos diarios durante 6 meses, que me dejan cansadísima, y posponer la cirugía para corregir el reflujo porque: "esta patología en adultos no es operable hasta un grado máximo porque es una intervención compleja que conlleva múltiples riesgos".

Durante los primeros meses tenía con frecuencia fiebre de $37^{\circ}$, pero al cabo de un tiempo, la febrícula se convierte en fiebre de entre $37^{\prime} 7^{\circ}$ y $38^{\prime} 5^{\circ}$, que se mantiene a diario y comienzo a perder kilos que no me sobraban pues mido 1'73 m y mi peso oscilaba entre los $62-65$ kilos. Tuve que dejar de trabajar, pues no podía hacer una vida normal.

Entonces mi marido, que es médico, decide llevarme a los internistas de un Hospital, a los que estoy muy agradecida, que después de muchísimos análisis y pruebas (hemocultivos, TAC, PET, etc...), descartan muchas otras enfermedades: infecciosas, tumorales, auto inmunes, etc... Durante este estudio los internistas se centran en el cansancio, la pérdida de peso y la fiebre, descartando que los síntomas pudiera causarlos el reflujo vesicoureteral. Un cardiólogo también me hizo un estudio completo.

Pero yo sigo con mi "fiebre de origen desconocido" sintiéndome cada vez más enferma, perdiendo peso hasta llegar a los 47 kilos.

Durante este período sufro cada vez más a menudo en el momento de ir a orinar porque durante la micción siento como si me clavaran un punzón desde la parte baja del vientre, en todo el trayecto del uréter en dirección al riñón, y al final de la micción permanecía un dolor intenso en el riñón durante 5 a 10 minutos acompañado de escalofríos. 
Esto sucede cada vez con más frecuencia, independientemente de que tuviera o no una infección urinaria, hasta tal punto que cuando sentía la necesidad de orinar pensaba: "otra vez tengo que pasar por esto".

Las molestias en ambos riñones se hicieron continuas, y por supuesto la fiebre, que se mantenía alrededor de los $38^{\circ}$, con lo que al final y después de casi 5 años desde que empezó el primer síntoma decido volver a visitar a un urólogo por recomendación de uno de los internistas. En el Hospital me propusieron cirugía a cielo abierto, sin ningún tipo de garantía de solucionar mi problema. Por ello decidí solicitar una segunda opinión, que me proporcionó el Dr... que me explica la existencia de una técnica minimamente invasiva que corrige el reflujo Vésico-ureteral, pero que en donde vivo sólo la realizan en urología pediátrica. ¡Yo tenía 33 años! pero el Dr... accedió a verme y a tratarme.

Mediante cistoscopia me puso unas inyecciones en la vejiga. Todo duro una media hora y me fui a mi casa el mismo día.

A los pocos días la fiebre empezó a remitir, ya no sentía ningún dolor renal y poco a poco empecé a encontrarme muchísimo mejor.

Unos meses después me hicieron un nuevo CUMS en el que se apreció que la operación había corregido el reflujo. Nunca más tuve dolores en los riñones ni fiebre. Fuírecuperando mi peso. Volví a mi trabajo. Hasta me quedé embarazada.

En estos momentos tengo 37 años, peso 60 kilos, tengo 2 hijos maravillosos y no sé por donde transcurren mis 3 uréteres porque no me duelen absolutamente nada.

Después de 4 años no he vuelto a padecer ningún tipo de infección urinaria ni nada que me recuerde mi reflujo vésico-ureteral bilateral.

Gracias Dr... por haber hecho que mi calidad de vida sea estupenda".

\section{DISCUSIÓN}

El RVU 1 tiene una tendencia a la desaparición espontánea sobre todo en grados bajos durante los primeros años de vida. Es más difícil la desaparición espontánea en RVU mayores de grado II, sobre todo si son bilaterales, y mayores de los 5 años (1).

Se calcula que el tiempo medio de desaparición del RVU 1, en los casos en que desaparece, es de $2 \%$ años, y que el $25 \%$ de los RVU 1 \% llegan a la adolescencia y persisten en la vida adulta (13).

Son escasas las publicaciones sobre el RVU $1^{\circ}$ en el adulto. La mayoría están relacionadas con la ITU (3, $9,14)$ sobre todo con el embarazo y las posibles consecuencias para el feto y la gestación (15-18).
Durante el embarazo los episodios de pielonefritis aguda son mas frecuentes en las pacientes con RVU (1517). La ITU es una de las causas más frecuentes de morbilidad materna durante el embarazo y el RVU y la nefropatía de reflujo predisponen a padecer pielonefritis gestacional (15-17).

Se conoce que el inicio de la vida sexual y el embarazo favorecen la aparición de ITUs en el sexo femenino. También el RVU favorece la ITU que desencadena pielonefritis ascendentes que pueden ocasionar daño renal en forma de Nefropatía de RVU.

El-Khatib y cols. (5) refieren que el RVU diagnosticado por primera vez en la vida adulta con frecuencia ocurre por el estudio de ITU; en esos pacientes la edad de inicio de las ITUs varía según el sexo: en el femenino suele ser entre los 15-30 años, mientras que en el masculino las primeras ITUs suelen ser antes de los 15 años.

Otros motivos de diagnóstico en el adulto son: hipertensión, insuficiencia renal, proteinuria, hematuria, dolor en flanco o litiasis $(3,4,6,9,11)$.

Otro motivo de diagnóstico es la disfunción del tracto urinario inferior (TUI). Virseda y cols. encuentran RVU en 19 adultos de 236 (8.1\%) estudiados con video urodinámica por síntomas de disfunción del TUI (19).

Aunque la incidencia del RVU disminuye con la edad su diagnóstico es importante ya que se encuentra RVU en el $10 \%$ de los adultos con insuficiencia renal terminal (20). Con el diagnóstico y tratamiento adecuado y precoz se podían prevenir o disminuir las complicaciones secundarias al RVU, tales como el fallo renal (20). El caso descrito ayuda a conocer más acerca de la morbilidad del RVU 1 durante la vida adulta demostrando que puede ser una enfermedad crónica sintomática, con importante afectación general y de la calidad de vida laboral y personal.

La morbilidad del RVU $1^{\circ}$ no se debe limitar a la hipertensión, proteinuria e insuficiencia renal. Debe considerarse además la pielonefritis crónica, la fiebre y al dolor renal y ureteral que, como esta paciente manifiesta, suponen un sufrimiento diario.

Es muy interesante el dolor tan claramente identificado como "dolor de reflujo", por la paciente, con las características mencionadas de: diario, agudo, intenso, lacerante, de 15-20 minutos de duración, que aumentaba durante la micción para desparecer 5-10 minutos después de la misma.

Personalmente conocemos otro caso del sexo masculino de 38 años de edad que nos consultó por el mismo tipo de dolor bilateral cuando retiene la orina mas de 5-6 horas. En su caso no se acompaña de pielonefritis crónica ni de fiebre. Destaca el antecedente de RVU y pielonefritis crónica febril, diagnosticado y no tratado durante la infancia. A los 12-13 años desaparecieron las ITUs febriles, y nunca más fue estudiado ni controlado por el RVU (no recuerda grado ni lado). Es médico cirujano y no ha querido someterse a una evaluación del RVU y sus posibles secuelas 
renales. Se conforma con orinar frecuentemente para evitar el "dolor del reflujo" de "intolerable intensidad, aunque de 5-10 minutos de duración". Por falta de más datos objetivos este caso no se incluye en el trabajo.

El enfoque terapéutico del RVU en el adulto es diferente que en el niño por que no ocurre la desaparición espontánea con el crecimiento (2). La PA puede ser necesaria toda la vida, aunque hay series de adultos con RVU observados sin antibióticos; parece que evolucionan mejor los del sexo masculino $(4,6,7)$. La evolución con ITUs febriles importantes es más rara y ocurre fundamentalmente en el sexo femenino (2). El tratamiento quirúrgico sirve para la erradicación de síntomas y poder suspender la PA $(6,8)$.

El TE minimamente invasivo parece ser la primera opción terapéutica por los buenos resultados con menores complicaciones y molestias postoperatorias que la cirugía abierta. Se ha publicado un $100 \%$ de éxito con TE en 9 mujeres adultas con RVU y pielonefritis (21).

\section{CONCLUSIONES}

Más que conclusiones, al tratarse de un caso clíni$\mathrm{co}$, se exponen algunas consideraciones.

Pensamos que el RVU $1^{\circ}$ puede ser sintomático en el adulto, y que debe de investigarse en ambos sexos cuando hay:

- ITUs febriles de repetición

- Dolor del Reflujo

Si se confirma el RVU, el TE puede curar el RVU y su sintomatología, además de disminuir o erradicar las ITUs febriles, con un procedimiento minimamente invasivo, que logra buenos resultados con escasas complicaciones.

En caso de antecedente de RVU persistente desde la infancia, debe hacerse un seguimiento básico no invasivo: clínico ecográfico y analítico, cada 1-2 años sobre todo si hay nefropatía de RVU.

Si el RVU tiene repercusión clínica, el TE puede mejorar al paciente.

\section{BIBLIOGRAFIA y LECTURAS RECOMENDADAS ("lectura de interés $\mathrm{y}^{* *}$ lectura fundamental)}

*1. MIGUÉLEZ, C.; GARCÍA MÉRIDA, M.; RECOBER, A.: "Reflujo Vésico Ureteral". Libro de Residentes de la Asociación Española de Urología. Coordinador: CASTIÑEIRAS FERNÁNDEZ, J. Grupo ENE, gráficas Marte. 281:302, 2007.

2. GREENFIELD, S.P.; WAN, J.: "The diagnosis and medical management of primary vesicoureteral reflux". Pediatric Urology. Gearhart JP, Rink RC, Mouriquand PDE editors. WB Saunders Company, Philadelphia, pp 382,2001
3. McGOVERN, J.H.; MARSHALL, V.F.; "Reflux and pyelonephritis in 35 adults". J. Urol., 101: 668, 1969.

4. NATIV, O.; HERTZ, M.; HANANI, Y. y cols.: "Vesicoureteral reflux in adults: A review of 95 patients". Eur. Urol., 13: 229, 1987.

5. EL-KATIB, M.T.; BECKER, G.J.; KINCAID-SMITH, P.: "Reflux nephropathy and primary vesicoureteric reflux in adults". Q. J. Med., 284: 1241, 1990.

6. NEVES, R.J.; TORRES, V.E.; MALEK, R.S. y cols.: "Vesicoureteral reflux in adult. IV. Medical versus surgical management". J. Urol., 132: 882, 1991.

7. GUTHMAN, D.A.; MALEK, R.S.; NEVES, R.J. y cols.: "Vesicoureteral reflux in adult. V Unilateral disease". J. Urol., 146: 21, 1991.

*8. KOHLER, J.; TENCER, J.; THYSELL, H. y cols.: "Vesicoureteral reflux diagnosed in adulthood. Incidence of urinary tract infections, hypertension, proteinuria, back pain and renal calculi". Nephrol. Dial. Transplant., 12: $2580,1997$.

9. BERQUIST, T.H.; HATTERY, R.R.; HARTMAN, G.W. y cols.: "Vesicoureteric reflux in adults". AJR, 125: 314, 1975.

10. SENOH, K.; IWATSUBO, B.; MOMOSE, S. y cols.: "Non-obstructive Vesicoureteral reflux in adults: Value of conservative treatment". J. Urol., 117: 566, 1977.

*11. ZHANG, Y.; BAILEY, R.R.: “A long term follow up of adults with reflux nephropathy”. N. Z. Med. J., 108: 142, m1995.

12. CHOI, Y.D.; YANG, W.J.; DO, S.H. y cols.: "Vesicoureteral reflux in adult women with uncomplicated acute pyelonephritis". Urology, 66: 55, 2005.

13. GODLEY, M.L.: "Vesicoureteral Reflux: Pathophysiology and experimental studies". Pediatric Urology. Gearhart JP, Rink RC, Mouriquand PDE, Eds. WB Saunders, Philadelphia, pp 359-381, 2001.

14. SERVADIO, C.; SHACHNER, A.: "Observations on vesicoureteral reflux and chronic pyelonephritis". J. Urol., 103: 722, 1970.

15. JUNGERS, P.; HOUILLIER, P.; CHAUVEAU, D. y cols.: "Pregnancy in women with reflux nephropathy". Kidney International, 50: 593, 1996.

16. McGLADDERY, S.L.; APARICIO, S.; VERRIER-JONES, K. y cols.: "Outcome of pregnancy in an OxfordCardiff cohort of women with previous bacteriuria". Quarterly Journal of Medicine, 83: 533, 1992.

17. MARTINELL, J.; JODAL, U.; LIDIN-JANSON, G.: "Pregnancies in women with and without renal scarring after urinary infections in childhood". BMJ, 300: 840, 1990.

18. MANSFIELD, J.T. y cols.: "Complications of pregnancy in women after childhood reimplantation for vesicoureteral reflux: An update with 25 years of follow up". J. Urol., 154: 787, 1995.

19. VIRSEDA CHAMORRO, M.; DIZ RODRIGUEZ, R.; SALINAS CASADO, J. y cols.: "Urodynamic factors associated to vesicoureteric reflux in adults". Arch. Esp. Urol., 59: 702, 2006.

**20. BUCKLEY, O.; GEOGHEGAN, T.; O`BRIEN, J. y cols.: "Vesicoureteric reflux in the adult". Br. J. Radiol., 80: 392, 2007.

*21. OEZEKE, Z.; FROMER, D.; KATZ, M.H. y cols.: "Endoscopic management of vesicoureteral reflux in women presenting with pyelonephritis". J. Urol., 176: 2219, 2006. 